\title{
Transient Analysis of Air/Oil Two-Phase Flow in Bearing Chamber under the Periodic Boundary Conditions
}

\author{
Wang Fei, Guoding Chen, Yang Fu and Wang Tao \\ School of Mechanical Engineering, Northwestern Polytechnical University, Xi'an 710072, China
}

\begin{abstract}
The air/oil two-phase flow is extremely complex in the aero-engine bearing chamber, so the comprehensive understanding of the flow phenomenon is helpful to improve the design of the lubrication and heat exchange system. In this paper, transient CFD simulation is developed to analyse the air/oil two-phase flow in the bearing chamber. The velocity and temperature distributions of air and oil with time and space are discussed. The results show that under the periodic conditions, the velocity and temperature of the air and oil in the bearing chamber present quasi periodic characteristics. And the velocity and temperature distribution of air/oil two-phase flow in bearing chamber is independent with time in the bearing chamber. The research work will contribute to understand the nonlinear characteristics of air/oil two-phase flow in the bearing chamber.
\end{abstract}

\section{Introduction}

Design of aero-engine lubrication system and secondary air flow system depends on the understanding of complicate air/oil two-phase flow in the bearing chamber. But lubricating oil and sealing air in the bearing chamber formed a complex air/oil two-phase flow, which results the design of the lubrication system is very difficult [1]. However, scholars have carried out a lot of research on the air/oil two-phase flow and the influence of different operating conditions in the bearing chamber.

Gorse et al. [2] measure the air phase velocity field in the bearing chamber by Laser Doppler Anemometry (LDA), analyse the effect of the bearing chamber air phase swirl on the flow field distribution, and discuss the influence of different operating conditions on air phase velocity distribution. Aroussi et al. [3] measure the velocity of oil droplets and air in the bearing chamber by PIV technology, and analyse the effect of rotor speed on the movement velocity of oil droplets. Gorse et al. [4] use three-dimensional particle image velocimetry (SPIV) technology in the bearing chamber test rig to reveal the oil droplet generation mechanism of the cylindrical roller bearing and carry out experiment research, probes into the influence of bearing support structure of oil droplets flow model, and analysis the influence of rotor speed, fuel supply and flow rate on the oil droplet velocity. Rodkey et al. [5] describe the flow characteristics of oil film on the wall of the bearing chamber, and analyse the effect of rotor speed and flow rate on the oil film thickness. Chen Guoding et al. [6] discuss the effect of the structure parameters of bearing chamber and working conditions on flow patterns, reveal the pressure and velocity distribution laws under different rotating speed.
Ao Wenwei et al. [7] utilize CFD software to simulate the air/oil two-phase flow in the bearing chamber, and analyses the influence of operating conditions on the flow state of the lubricating medium.

But these research works mainly focus on the constant air and oil supply, did not consider the influence of varying air and oil supply on the velocity and temperature of air/oil two-phase flow in the bearing chamber. However, in practical engineering, the air and oil supply is not constant, but change with time for special purpose. Therefore, the periodic air and oil supply with time was considered in this paper. Air/oil two-phase flow phenomena in the bearing chamber was simulated. Firstly, the geometric model of air/oil two-phase flow was built and then meshed. Secondly, the transient CFD simulation was completed by setting period boundary conditions of air and oil supply. Then the velocity and temperature field distribution of air/oil two-phase flow was analysed. Finally, the change of velocity and temperature of air/oil two-phase flow with time and space position was discussed. This work contributes to understand the nonlinear problem of air/oil two-phase flow in the bearing chamber.

\section{Bearing chamber structure and air/oil physical properties}

Figure 1 shows the basic structure of the bearing chamber, and table 1 lists its main structural parameters. The lubricating oil is thrown into the bearing chamber by the bearing retainer, then oil film is formed on the surface of the wall. Sealing air flows into the bearing chamber through the seal clearance. Lubricating oil and sealing air 
in the bearing chamber form a complex air/oil two-phase flow.

The physical properties of lubricating oil are described as follow: oil density $\rho_{L}=954 \mathrm{~kg} / \mathrm{m}^{3}$, dynamic viscosity $\mu_{L}=9.5 \times 10^{-3} \mathrm{~Pa} \cdot \mathrm{s}, \quad$ specific heat capacity $C_{L}=2000 \mathrm{~J} /(\mathrm{kg} \cdot \mathrm{K})$, thermal conductivity rate $\lambda_{L}=0.15$ $\mathrm{W} /(\mathrm{m} \cdot \mathrm{K})$, and the surface tension coefficient $\sigma_{L}=0.035$ $\mathrm{N} / \mathrm{m}$; The physical properties of seal air are described as follow: air density $\rho_{g}=2.92 \mathrm{~kg} / \mathrm{m} 3$, dynamic viscosity $\mu_{g}=1.84 \times 10^{-5} \mathrm{~Pa} \cdot \mathrm{S}$, specific heat capacity $C_{g}=1004.4$ $\mathrm{J} /(\mathrm{kg} \cdot \mathrm{K})$, thermal conductivity rate $\lambda_{g}=0.0261 \mathrm{~W} /(\mathrm{m} \cdot \mathrm{K})$.
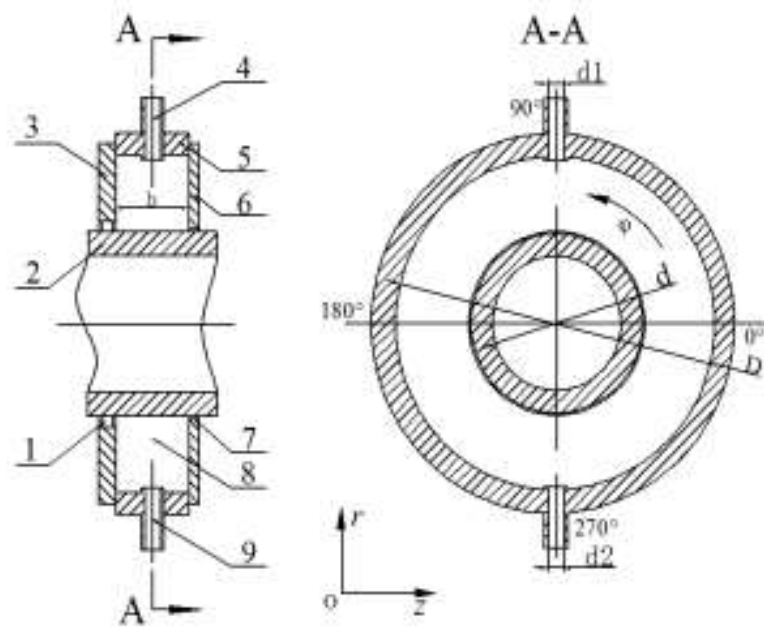

1-bearing rolling body; 2-rotator; 3-bearing seat; 4-vent pipe; 5chamber wall; 6-end cover; 7-seal clearance; 8-bearing chamber; 9 -scavenge pipe

Figure 1. Schematic of bearing chamber.

Table 1. The main structural parameters of the bearing chamber.

\begin{tabular}{|c|c|}
\hline Parameter & value \\
\hline Bearing chamber diameter D (mm) & 180 \\
\hline Bottom Rotor diameter d (mm) & 124 \\
\hline Bearing chamber width b (mm) & 20 \\
\hline Vent pipe diameter d1 $(\mathrm{mm})$ & 15 \\
\hline Scavenge pipe diameter d2 (mm) & 15 \\
\hline
\end{tabular}

\section{Mathematical model}

Lubricating Oil deposits on the wall of the bearing chamber, and the air/oil two-phase flow states of the air and oil film is formed. The equations of continuity, momentum and energy conservation equations are used to describe the air/oil two-phase flow state in the bearing chamber, and the expressions of the 3 governing equations are as follows:

$$
\begin{aligned}
& \frac{\partial\left(\gamma_{\alpha} \rho_{\alpha}\right)}{\partial t}+\nabla \cdot\left(\gamma_{\alpha} \rho_{\alpha} U_{\alpha}\right)=0 \\
& \frac{\partial\left(\gamma_{\alpha} \rho_{\alpha} U_{\alpha}\right)}{\partial t}+\nabla \cdot\left(\gamma_{\alpha} \rho_{\alpha} U_{\alpha} \otimes U_{\alpha}\right)=-\gamma_{\alpha} \nabla \mathrm{p}_{\alpha} \\
& +\nabla \cdot\left(\gamma_{\alpha} \mu_{\alpha}\left(\nabla U_{\alpha}+\left(\nabla U_{\alpha}\right)^{\mathrm{T}}\right)\right)+S_{M \alpha}+M_{\alpha} \\
& \frac{\partial\left(\gamma_{\alpha} \rho_{\alpha} H_{\alpha}\right)}{\partial t}-\gamma_{\alpha} \frac{\partial p}{\partial t}+\nabla \cdot\left(\gamma_{\alpha} \rho_{\alpha} U_{\alpha} H_{\alpha}-\gamma_{\alpha} \lambda_{\alpha} \nabla T_{\alpha}\right) \\
& -\nabla \cdot\left(\gamma_{\alpha} \mu_{\alpha}\left(\nabla U_{\alpha}+\left(\nabla U_{\alpha}\right)^{\mathrm{T}}-\frac{2}{3} \nabla U_{\alpha} \delta\right) U_{\alpha}\right)=S_{\alpha}+Q_{\alpha}
\end{aligned}
$$

where $\rho, \mu$ and $\lambda$ is respectively for the density, viscosity and thermal conductivity of lubricating oil or sealing air; $\gamma$ is volume fraction; $U$ is the fluid velocity vector; $T$ is the fluid temperature; $t$ is time; $\mathrm{p}$ is pressure; $S_{M}$ is momentum source terms; $S$ is heat source; $M$ is interphase drag force; $Q$ is interphase heat exchange; $H$ is total energy; the subscript $\alpha$ respectively denotes the lubrication oil when $\alpha$ equals to $L$, and sealing air when $\alpha$ equals to $g$. The units of varitions in the formula adopt internationally standard units.

\section{Numerical model}

\subsection{Geometry model and mesh}

According to the structure of the bearing chamber as shown in Figure 1, the three dimensional geometry model is built, and the ICEM software is used to mesh the geometric model. Hexahedron element is employed to divide the computational domain. Considering air swirl flow near the wall, the region grids are refined. The total number of grids is 324948. The quality of the grid is judged by the quality criterion. The whole mesh quality is more than 0.3 , which meets the requirements of the calculation.

\subsection{Boundary and initial conditions}

In order to achieve the transient CFD simulation of air/oil two-phase flow in the bearing chamber, the applied boundary conditions include inlet and outlet boundary conditions and wall surface conditions. The the boundary conditions set as follows:

(1) inlet boundary conditions: the periodic flow rate with time. The sealing air varies in the range of $2.5 \sim 3.5 \mathrm{~g} / \mathrm{s}$, time period is 3 seconds; the lubricating oil rate varies in the range of $0.63 \sim 3.14 \mathrm{~L} / \mathrm{min}$, time period is 3 seconds; the temperature of sealing air is $450 \mathrm{~K}$, where the temperature of lubricating oil is $330 \mathrm{~K}$; the function expressions of sealing air mass flow rate and lubricating oil volume flow rate were written by CEL language, then implemented on the vent port and the scavenge port. The operate temperature is $330 \mathrm{~K}$.

(2) outlet boundary conditions: the relative pressure of the vent port and the scavenge port is 0 ; the environment temperature of the vent port is $540 \mathrm{~K}$; 
(3) wall boundary conditions: no slip wall boundary condition implements on the rotor surface, and the rotor speed is $5000 \mathrm{rpm}$; bearing chamber wall surface sets no slip stationary wall, and initial wall oil film thickness is $1 \mathrm{~mm}$.

\subsection{Computing method}

The Simple algorithm is used to solve the continuity equation, momentum equation and energy conservation equation and turbulent equation. The calculation step sets 120 , and the convergence criterion is $1 \times 10^{-4}$.

\section{Results and discussion}

\subsection{The change of the velocity and temperature of air/oil two-phase flow with time}

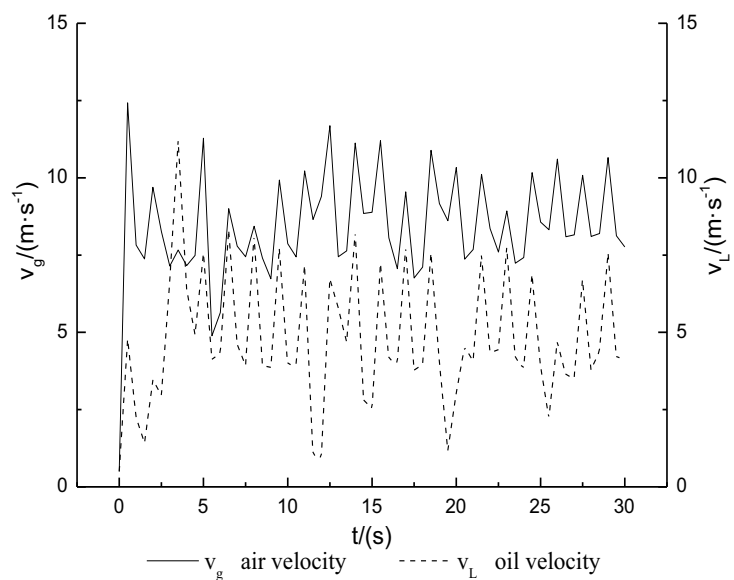

(a). The change of air and oil velocity with time

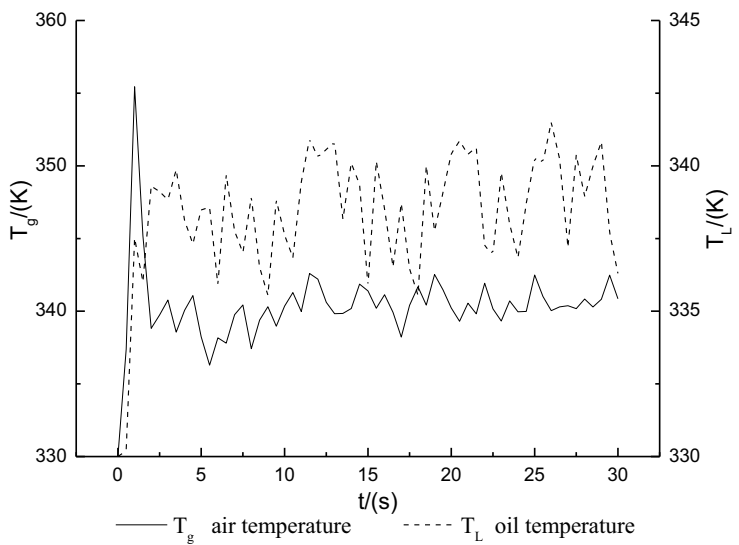

(b). The change of air and oil temperature with time

Figure 2. The change of velocity and temperature with time.

Figure 2 shows the velocity and temperature of the air phase at the point $(0.075,0,0.01)$, and the velocity and temperature of the oil at the point $(0.088,0,0.01)$ in the bearing chamber. The curves of the air velocity and the oil velocity have similar feature, which all show strong fluctuation, but the difference between the air temperature and the oil temperature is obvious. Figure 2 (a) shows the change of the air and oil velocity with time. During the initial period, the variation of air velocity is larger than the variation of oil velocity, and the distributions of air and oil velocity did not present period changes. But with time increasing, the air/oil two-phase flow in the bearing chamber tends to stable, and the velocity distributions of air and oil shows a quasiperiodic change. The reason is that periodic air mass flow rate and periodic oil volume rate were given as initial conditions, but a large number of oil droplets and the secondary oil droplets exist in the bearing chamber, which results that the velocity distributions of air and oil change with time at the same position. Figure 2 shows the change of the air and oil temperature with time. In the initial time, because the flow of air and oil in the bearing chamber has not reached a steady state, the air and oil temperature fluctuation is fierce. But with time increasing, the fluctuation of air and oil temperature tends to stable in a certain temperature range. Due to the smaller oil volume fraction, so the temperature range of oil is larger than air, the period feature of oil is not obvious. In general, the temperature and velocity of air and oil in the bearing chamber appear a periodic variation with time due to periodic air mass flow and oil volume flow.

\subsection{The change of the velocity and temperature of air/oil two-phase flow with space}

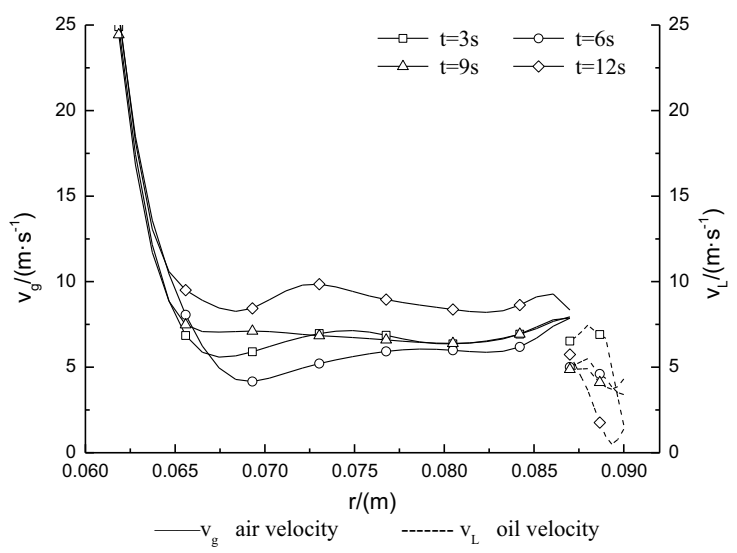

(a). The change of the air and oil velocity along the radial direction.

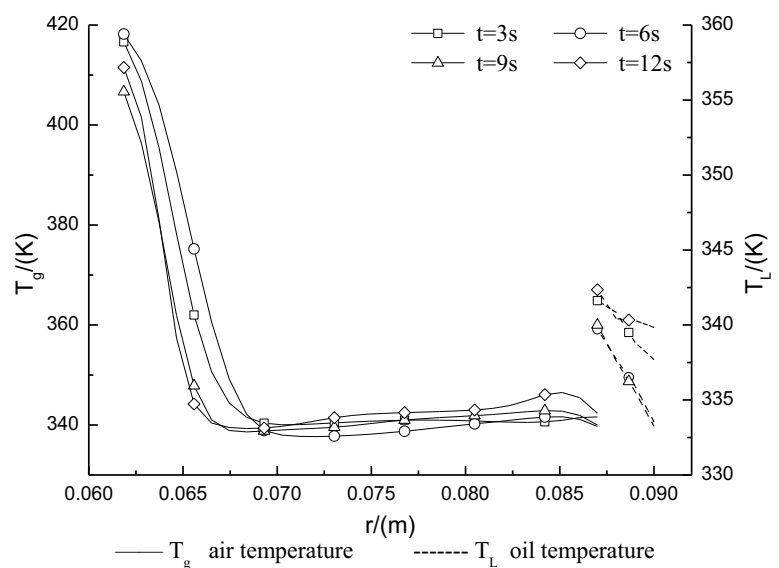

(b). The change of the air and oil temperature along the radial direction.

Figure 3. The change of velocity and temperature along the radial direction. 
Figure 3 shows the change curves of velocity and temperature of air and oil at four different times along the radial direction in the bearing chamber. Influenced by rotor speed, the velocity and temperature gradient near the rotor surface is larger, but the velocity and temperature gradient of the air and oil has little change at different locations of the bearing chamber core area. Figure 3 (a) presents the change of the air phase and oil phase velocity at different times along the radial direction.

It shows that the air and oil phase velocity did not appear periodicity in the radial direction of the bearing chamber. In the vicinity of rotor surface, the air velocity phase is higher than other region, and the velocity gradient is larger, but the velocity gradient is not great in the bearing chamber core area. The oil film of bearing chamber wall is affected by the boundary layer, so the velocity gradually decreases along the radial direction. Fig. 3 (b) gives the variation curve of the air phase and oil phase temperature at four different times along the radial direction in the bearing chamber. The air and oil temperature in the radial direction did not show a period change. In the radial direction, the air temperature near the rotor surface is higher than the core area of bearing chamber. In the core area, the temperature distribution of air phase is relatively uniform, and at the different times the changes are tiny. And the oil temperature changes in a certain range at the different times. The reason is that period oil supply makes the heat transfer of oil volatile.

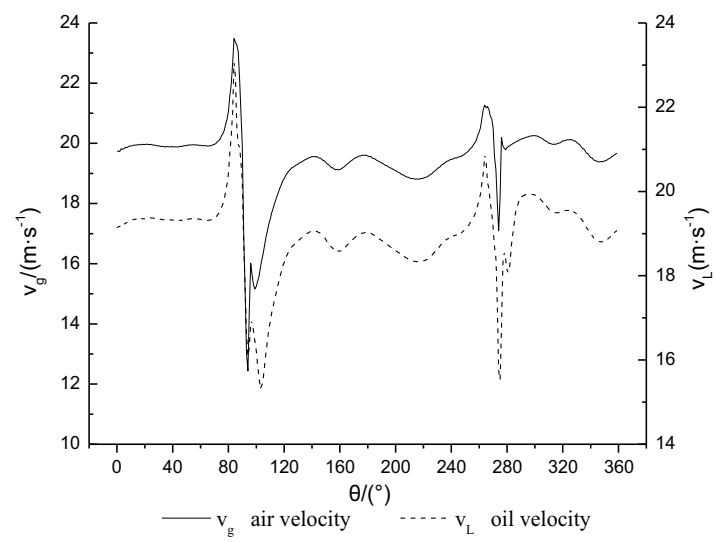

(a). The change of the air and oil velocity along the circumferential direction.

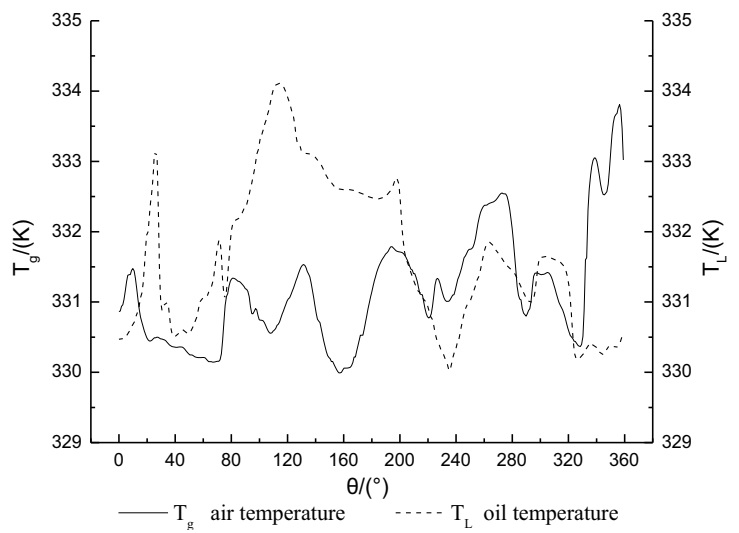

(b). The change of the air and oil temperature along the circumferential direction.

Figure 4. The change of velocity and temperature along the circumferential direction.
Figure 4 shows the velocity and temperature of the air at $\mathrm{t}=12 \mathrm{~s}$ along the circumferential direction $\mathrm{r}=0.75 \mathrm{~m}$, and the velocity and temperature of the oil along the $r=0.88 \mathrm{~m}$ circumferential direction. As can be seen from the figure, the velocity field of air and oil in the circumferential direction of the bearing chamber is similar to the velocity field. The temperature field is more complicated than the velocity field. Figure 4 (a) shows the air and oil velocity along the circumferential direction at $\mathrm{t}=12 \mathrm{~s}$. Due to the existence of the vent port and the scavenge port, the velocity of the air and oil leaps in these regions, but the velocity is relatively stable in the other regions of bearing chamber. The air velocity distribution is similar with oil velocity distribution. Figure 4 (b) gives the change of air and oil temperature in the bearing chamber along the circumferential direction at $\mathrm{t}=12 \mathrm{~s}$. As seen in the figure the change of air and oil temperature along the circumferential direction is more complex. The highest air temperature is near the vent. Because environment temperature near the vent is large. Influence by the wall shear stress, the lubricating oil accumulates at the lower right part in bearing chamber, so temperature is higher.

\section{Conclusions}

With the velocity and temperature distributions of the air/oil two-phase flow in the bearing chamber show an approximate periodicity with time, but the velocity and temperature distribution of the air/oil two-phase flow in the bearing chamber did not show obvious periodicity with space. The research results in this paper contribute to the understand and study the periodic and nonlinear problems of air/oil two-phase flow in the bearing chamber.

\section{Funding}

This study is supported by the National Natural Science Foundation of China (grant number 51275411).

\section{References}

1. C. Guoding, C. Bo, W. Jun, C. Chen, J. Mech. Eng, 139 (2011).

2. P. Gorse, K. Willenborg, S. Busam, J. Ebner, K. Dullenkopf, S. Wittig, Proc. ASME T. Expo, 16 (2003).

3. A. Aroussi, G. Ishaq, M. Menacer, Proc. ASME Joint Fluids Eng. Conf, 6 (2003).

4. P. Gorse, K. Dullenkopf, H. J. Bauer, S. Wittig, Proc. ASME T. Expo, 9 (2008).

5. S. C. Rodkey, Heister S D, S. H. Collicott, 43rd AIAA/ASME/SAE/ASEE Joint Prop. Conf, 8(2007).

6. C. Guoding, C. Bo, L. Yajun, C. Chen. J. Nor. Pol. Uni, 62 (2011).

7. A. Wenwei, S. Hua, C. Guoding. Mech. Sci. Tech. Aero. Eng, 1064 (2009). 